International Journal of Cardiology and Research (IJCRR)

ISSN 2470-4563

\title{
Takotsubo Syndrome Triggered by Acute Coronary Syndrome in a Cohort of 20 Patients: An often Missed Diagnosis
}

Research Article

Y-Hassan S

Department of Cardiology, Karolinska Institute at Karolinska University Hospital, Sweden.

\section{Abstract}

Background: In spite of being a major stress factor, acute coronary syndrome (ACS) is still regarded as an exclusion criterion for takotsubo syndrome (TS). The aim of the study is to find out an association between ACS, post-ischemic myocardial stunning (PIMS) and TS.

Methods: A series of twenty patients with ACS having an acute coronary culprit lesion on coronary angiography and signs of left ventricular dysfunction, which extended beyond the supply region of the diseased coronary artery were studied.

Results: Thirteen (65\%) out of the 20 patients were women. Ages ranged from 37 to 85 years old. All patients had signs consistent with PIMS. The extent of the stunned myocardium in all patients went beyond the supply region of the diseased coronary artery. The left ventricular myocardial stunning had also a peculiar ballooning appearance during systole, a clinical picture and course also consistent with TS. In all patients, there was no evidence of any emotional or physical stress factor apart from the ACS event.

Conclusions: PIMS induced by ACS had typical feature of TS suggesting that PIMS and TS may be two (among other) different names for the same acute cardiac clinical condition. ACS may trigger rather than exclude TS.

Keywords: Takotsubo; Stunned Myocardium; Acute Coronary Syndrome; Myocardial Infarction; Broken Heart Syndrome.

\section{*Corresponding Author:}

Shams Y Hassan MD,

Department of Cardiology, Karolinska University Hospital, Huddinge,

Department of Cardiology, S- 14186 Stockholm, Sweden.

Tel: +46858582805

Fax: +46 858586710

E-mail: shams.younis-hassan@karolinska.se

Received: May 28, 2015

Accepted: June 16, 2015

Published: June 22, 2015

Citation: Y Hassan S (2015) Takotsubo Syndrome Triggered by Acute Coronary Syndrome in a Cohort of 20 Patients: An Often Missed Diagnosis. Int J Cardiol Res. 02(2), 28-33. doi: http://dx.doi. org/10.19070/2470-4563-150007

Copyright: Y-Hassan S ${ }^{\odot}$ 2015. This is an open-access article distributed under the terms of the Creative Commons Attribution License, which permits unrestricted use, distribution and reproduction in any medium, provided the original author and source are credited.

\section{Introduction}

Post-ischemic myocardial stunning (PIMS) is defined as viable myocardium that exhibits prolonged left ventricular dysfunction after resolution of a discrete but transient episode of ischemia. PIMS has been identified following reperfusion, angioplasty, unstable angina, exercise induced angina, ischemic stress induced by dobutamine or dipyridamole, myocardial infarction and cardiac surgery $[1,2]$. Takotsubo syndrome (TS) is characterized by a clinical and electrocardiographic (ECG) presentation resembling that of acute coronary syndrome (ACS). A peculiar circumferential transient ventricular wall motion abnormality resulting in a conspicuous ballooning of the left ventricle during systole is regarded as the footprint of the disease $[3,4]$. Countless emotional and physical stress factors may trigger TS $[5,6]$. Obstructive coronary artery disease and ACS are still regarded as exclusions diagnostic criteria for TS [7]. In this report, up to my knowledge for the first time, a series of 20 patients with ACS having an acute coronary culprit lesion and triggering TS is presented. Furthermore, substantial literature data supporting the concept that PIMS and TS are two different names for the same clinical condition are provided.

\section{Methods}

Over a period of 3 years (May 2010 to May 2013), 20 cases were identified in our department with the following clinical characteristics: First, a clinical presentation of ACS; second, a coronary angiography, done during the first days of admission, showing an acute coronary culprit lesion accounting for the ACS; third, left ventriculography showing signs of left ventricular dysfunction, which extended beyond the supply region of the diseased coronary artery; Fourth, 18 patients have followed up with some forms of cardiac image study to demonstrate the reversibility of the left ventricular dysfunction. The study is an observational one and the left ventriculography was done either when the coronary 
culprit lesion was not obvious at the instant of undertaking coronary angiography as that demonstrated in Figure 1 or done by this author when left ventriculography deemed not to be a burden on the clinical condition of the patient. Left ventriculography was not done systematically in all patients with acute coronary syndrome. The study was approved by the local institutional review board and informed consent was obtained from all survived pa- tients.

\section{Results}

A series of 20 patients with ACS and left ventricular dysfunction were included in the study. Some clinical, angiographic findings, type of therapy, TS localization and the course of LVWMA are shown in Table 1 . Thirteen $(65 \%)$ of the patients were women.

Table 1. Some clinical and angiographic findings of the 20 patients.

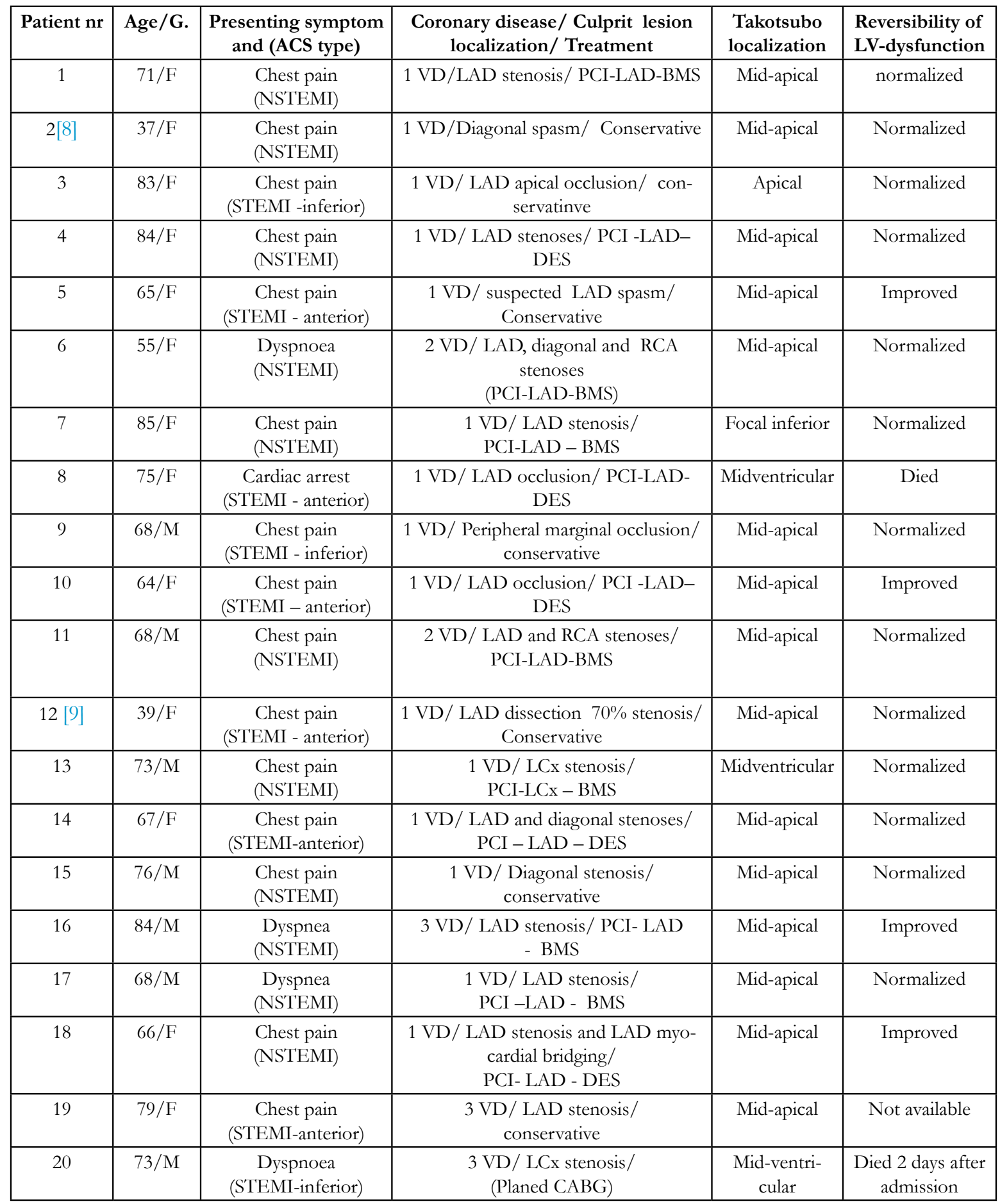

ACS, acute coronary syndrome; LV, left ventricular; M, male; F, female; STEMI, ST elevation myocardial infarction; NSTEMI, non ST elevation myocardial infarction; LAD, left anterior descending artery; LCx , left circumflex artery; RCA, right coronary artery; BMS, bare metal stent; DES, drug-eluting stent; PCI, percutaneous coronary intervention; Refs, reference; 1 V, one vessel disease; 2 VD, two vessel disease; $3 \mathrm{VD}$, three vessel disease. 
Ages ranged from 37 to 85 years old (mean age 69 years). All of the patients presented with a clear clinical picture of ACS and had a coronary culprit lesion accounting for the ACS. There was no history of chronic ischemic heart disease or previous ACS events. The presenting symptom was chest pain of cardiac ischemic nature in 15 patients, dyspnoea in 4 patients and cardiac arrest preceded by chest pain in one patient. Eleven patients had ECG changes of non-ST-elevation myocardial infarction (NSTEMI). Nine patients had ECG signs of ST elevation myocardial infarction (STEMI) (6 anterior, 3 inferior). There was mild to moderate elevation of myocardial infarction biomarkers in 12 patients $(10$ patients with NSTEMI and 2 patients with STEMI) and marked elevation in 8 patients ( 7 patients with STEMI and one patient with NSTEMI). Two patients had coexistent atrial fibrillation. Six patients developed signs of heart failure, which was treated successfully. Two patients died during the index presentation, the first patient who presented with cardiac arrest died one week after admission because of hypoxic brain injury, the second patient died 2 days after admission because of cardiac rupture. All other patients improved without recurrence of TS.

\section{The Coronary Artery Lesions}

Fifteen of the patients had one-vessel disease, 2 patients had twovessel disease and 3 patients had 3-vessel disease. The coronary culprit lesion was a tight stenosis in the left anterior descending artery (LAD) in 11 patients, where one deemed to have spasm in a long segment. Three patients had LAD occlusion: one presented with cardiac arrest; one caused by LAD dissection distal to a stent, which was implanted one hour before during the same day and the third one had apical LAD occlusion. One patient had spontaneous LAD dissection confirmed by intravascular ultrasound examination. One patient had spasm in a large diagonal branch, which was confirmed by follow up angiography, and one patient had a tight diagonal stenosis. Three patients had acute coronary lesions in the left circumflex artery: one patient with stenosis in the proximal part of the left circumflex artery; the second one with a peripheral obtuso-marginal branch occlusion; and the third patient had peripheral stenosis in a dominant left circumflex artery. Intracoronary nitroglycerin injection was given to all patients with coronary stenosis including the two patients with suspected coronary spasm with no remarkable effect. Twelve patients were treated with percutaneous coronary intervention with stent implantation. Conservative treatment was selected in 7 patients (two patients with suspected coronary spasm, two patients with very peripheral occlusive lesions, one with spontaneous LAD dissection, one patient with diagonal stenosis and one patient with advanced coronary artery disease.

\section{The pattern of the left ventricular wall motion ab- normality in the study}

All twenty patients had ACS documented clinically and with coronary angiography. The ischemic insult resulted in a prolonged left ventricular dysfunction, which was completely normalized in 13 patients. In the remaining 4 survived patients, there was resolution of the greater part of the left ventricular dysfunction. These features of ischemic-induced left ventricular dysfunction were consistent with PIMS. Nevertheless, the distribution of the myocardial stunning went beyond the supply region of the diseased coronary artery in all patients. Furthermore, the left ventricular wall motion abnormality (LVWMA) resulted in a conspicuous ballooning of the left ventricle during systole in 19 of the 20 patients. These features of LVWMA are also consistent with TS. The localization of the left ventricular ballooning or dysfunction was mid-apical in 15 patients, mid-ventricular in 3 patients, apical in one patient and focal (infero-apical) in one patient. Two representative patients with ACS inducing TS with figures have been shortly described. The first case is an acute LAD occlusion inducing mid-ventricular TS (Figure 1); the second case is an acute obtuso-marginal occlusion inducing mid-apical TS (Figure 2). Interestingly, the LVWMA was remote from the diseased coronary artery in 6 patients (as dem-

Figure 1. Case number 8 is a 75-year-old previously healthy woman developed cardiac arrest after an acute onset of severe chest pain. The patient was resuscitated and ECG examination showed acute anterior ST elevations. Because the left anterior descending artery (LAD) occlusion was not detected initially (A and B), left ventriculography was performed and showed signs of mid-ventricular takotsubo syndrome (TS) (C, diastole and D, systole). Further analysis of coronary angiography showed acute LAD occlusion (E, arrows) which was successfully recanalized and stented. The ballooning of the midventricular region of the left ventricle cannot be accounted by LAD occlusion. The patient was and remained unconscious,

which possibly may also be discussed as a likely trigger factor for TS. The patient received palliative therapy thereafter because she deemed to have permanent hypoxic brain damage and died one week after admission. Conceivably, the reversibility of the left ventricular dysfunction could not be determined. LCA, left coronary artery; RCA, right coronary artery.

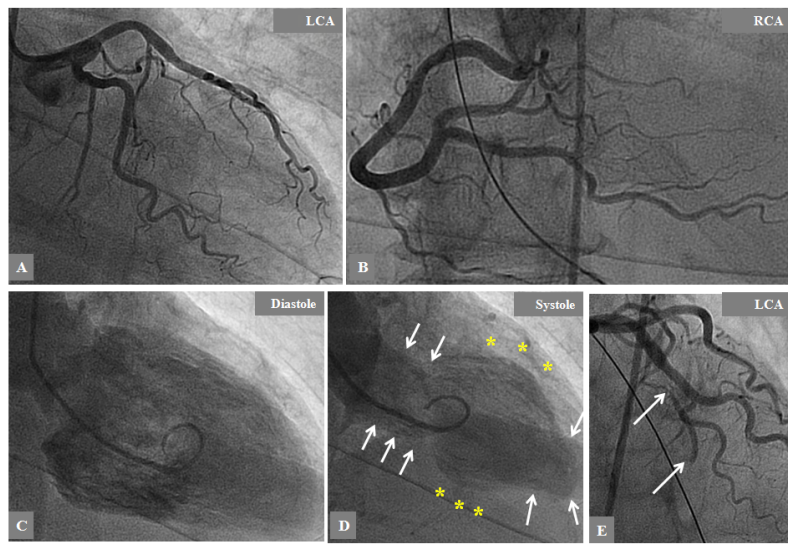


Figure 2. Case number 9 is a 68 -year old man with a history of bronchial asthma and paroxysmal atrial fibrillation. He was admitted because of 2 hours duration of severe chest pain. The ECG showed ST-elevation over the inferior leads and

ST-depressions anterolaterally. Coronary angiography showed normal right coronary artery and LAD. A non-occluding thrombus in the proximal part of the left circumflex artery was suspected and a peripheral occlusion of an obtuso-marginal branch (A and B) was seen. Left ventriculography showed a typical mid-apical type of TS (C and D), which could not be accounted by the diseased vessel. Echocardiography showed complete resolution of the mid-apical ballooning 4-5 hours after left ventriculography ( $E$ and $F)$.

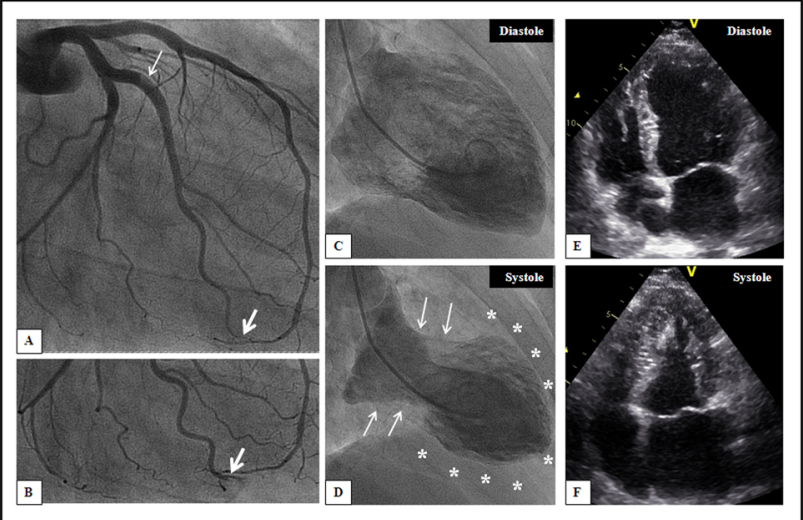

onstrated in both figure- 1 and figure-2) and extended beyond the territory region of the diseased coronary artery in the remainder. Follow up cardiac image studies done in 17 patients showed normalization of left ventricular function in 13 patients and marked improvement in 4 patients. In one patient with typical mid-apical ballooning, the follow-up cardiac image study was not available. Two patients died during the index presentation and follow up cardiac image study was understandably not done.

\section{Discussion}

The main findings in this series of 20 patients are that PIMS was induced in all patients presented with ACS and documented coronary culprit lesions. The myocardial stunning observed in these patients had also all the characteristic features of TS. As in other TS populations, the women predominated (65\%) in this cohort of patients $[5,6]$. Critical review of the history of the patients could not reveal any emotional or physical stress factor preceding the onset of the disease apart from the ACS incident. Consequently, PIMS in this series of 20 patients had the same features of TS and were triggered by ACS. This indicates that PIMS and TS are most probably manifestations of the same acute cardiac disease entity. Substantial literature evidences supporting this hypothesis are presented $[8,9]$.

\section{PIMS and TS have similar features}

The clinical presentation and course of the disease in PIMS and TS are identical $[2,4]$. TS typically presents with signs and symptoms resembling that of ACS [3]. The ACS is one of the most important "causes" of PIMS [2]. Both conditions result in severe transient LVWMA, which resolves completely during the ensuing days or weeks. The extent of myocardial dysfunction is disproportional to the mildly elevated myocardial infarction biomarkers in both PIMS and TS $[2,5,6]$. All of these features and course of the disease were seen in our patients. The evolutionary ECG changes and wide spread symmetrical T-wave inversion and prolonged QTc time occurring in TS are also typical electrocardiographic features in PIMS especially that induced by LAD stenosis [10]. Twelve of our 20 patients had $\mathrm{T}$ wave inversion and prolonged QTc time. Kurisu et al., [11] reported on the time course of ECG changes in 16 patients with TS and 15 patients with minimal anterior myocardial infarction who underwent reperfusions therapy within 24 hours of onset. They showed similar time course of ECG changes with T-wave inversion and prolonged QTc time occurring twice. Both groups showed also dramatic resolution of left ventricular dysfunction.

One of the important characteristic features of the PIMS is the positive response to inotropic agents. It has also been demonstrated that low dose dobutamine stress echocardiography improved the systolic left ventricular function through normalizing or improving the hypokinetic segments in TS patients [12]. Uchida et al., [13] studied 9 patients with TS with scintigraphy for myocardial perfusion, imaging of fatty acid metabolism and sympathetic nervous system. They found that the myocardial scintigraphy improves in line with recovery of wall motion and in a way similar to that happens in PIMS. The absence of late gadolinium enhancement with cardiac magnetic resonance imaging (MRI) in TS supports the hypothesis that TS is due to regional myocardial stunning as that of PIMS [14]. Ibrahim et al., [15] have reported on simultaneous positron emission tomography/MRI in a patient with mid-ventricular TS where fusion overlay of fluorodeoxyglucose-positron emission tomography and MRI showed signs compatible with myocardial stunning in the midventricular region. Contraction band is a constant and an important histopathological feature in patients with TS [16]. This type of lesion is also a well-recognozied histopathological finding in successful coronary reperfusion, which is one of the important "causes" of PIMS [17]. Consequently, the two conditions, PIMS and TS, have almost the same clinical presentation, evolutionary ECG changes, cardiac image and scintigraphic findings and clinical course. Interestingly, Dote and Sato, when they first introduced the term takotsubo in 1990 and 1991, described the condition as myocardial stunning due to multi-vessel coronary spasm $[3,18]$. This is very obvious in the title of the paper published by the investigators [Myocardial stunning due to simultaneous multivessel coronary spasms: a review of 5 cases] [3]. Accordingly, it will be reasonable to argue that the PIMS and TS may be two different names for the same acute cardiac clinical condition. There is substantial evidence supporting the hypothesis, which has been discussed elsewhere, that this acute cardiac clinical condition is most probably caused by the local cardiac sympathetic disruption with norepinephrine 
seethe and spillover $[19,20]$.

\section{The pattern of the left ventricular wall motion ab- normality in TS and PIMS}

The LVWMA in TS has a peculiar circumferential pattern. This aor hypokinesis of the affected parts of the left ventricle together with the hyperkinesis of the unaffected normal region result in a conspicuous ballooning of the left ventricle during systole. This feature has become the hoofmark of TS. The pattern of ventricular wall motion abnormality in PIMS has not been well described. It was first after the introduction of the descriptive term takotsubo, some investigators began to describe the silhouette of the left ventricle during systole in patients with TS and introduced new terms as ampulla, amphora, hawk's beak, artichoke, apical ballooning, inverted takotsubo and the like [3, 21]. Before the era of takotsubo, the majority of case reports of myocardial stunning triggered by acute non-cardiac diseases were also described as transient left ventricular dysfunction or ventricular wall motion abnormality similar to that that of PIMS. Careful review of those cases indicates that they actually had takotsubo syndrome [2, 2224]. In the present study, 17 out of 20 patients with PIMS, the left ventricular dysfunction had a pattern of reversible left ventricular ballooning similar to that of TS. During the last few years and with the increasing recognition of TS, there have been reports indicating that ACS may be a trigger factor for TS [8, 9, 25-28].

In two studies with relatively large number of patients with typical TS, cardiac MRI has revealed findings suggestive of myocardial infarction in some of the patients $[29,30]$. This may indicate that myocardial infarction has triggered TS. Chao et al reported on the pattern of left ventricular dysfunction induced by acute occlusion of the LAD. They found that $26 \%$ of patients, who presented with anterior STEMI because of acute LAD occlusion had left ventricular contraction pattern typical for TS. Interestingly, the majority of those patients were females [31, 32].

\section{Stress and acute coronary syndrome}

Countless physical stress factors have been reported to precipitate TS [4-6, 33, 34]. In the present patient population, there was no history of any emotional or physical stress factor apart from the ACS event, which is actually a major stress factor. The $26 \%$ of TS-like left ventricular ballooning in acute myocardial infarction caused by LAD occlusion in the study published by Chao et al., [31] is comparable to the results of the study reported by Park et al., [35] where they examined 92 consecutive patients who were admitted to the medical intensive care unit for non-cardiac acute physical illnesses. They demonstrated echocardiographic evidence of TS in $28 \%$ of these patients. It will be unreasonable not to believe that the stress of an ACS and other acute cardiac diseases could also trigger TS.

\section{The implications of the study}

Absence of obstructive coronary artery disease and angiographic evidence of acute plaque rupture are still regarded as exclusions criteria in all the existing diagnostic criteria for TS [36]. It has been reported that 1-2 percent of patients undergoing coronary angiography because of ACS have TS [4-6]. The main reasons for the under-diagnosed TS in patients with ACS is the existing expert consensus document on the definition of acute myocardial infarction and the current diagnostic criteria for TS [36, 37]. The present Diagnostic Criteria for both TS and myocardial infarction have blocked identification of TS triggered by ACS. This is well demonstrated in the recently reported paper by Prasad et al., [38] The authors reported a very low incidence $(0,5 \%)$ of TS in HORIZON-AMI trial in those patients who were examined by left ventriculography. The study included 2648 patients presenting with STEMI and were examined by both coronary angiography and left ventriculography at the most appropriate time for identification of patients with TS. The strikingly low incidence of TS in that report is attributed, in my opinion, to the use of the present diagnostic criteria for TS. In those criteria, an angiographic evidence of acute plaque rupture is an exclusion criterion, which immediately resulted in disqualification of 2636 patients to revise for signs of TS alongside in that report. Our study and with the support of the literature data provide a clear-cut evidence that ACS is an important trigger factor for TS. This indicates that the current diagnostic criteria for TS need to be revised.

The second important point, which should be considered, is the impact of ACS-triggered TS on the management of patients with ACS. This indicates that in addition to the conventional treatment of ACS, further steps should be directed to the pathogenesis of ACS induced TS. Regarding the etiopathogenesis of TS, two main pathologic mechanisms are under discussion for the current time. The first one is the coronary ischemia (multi-vessel coronary spasm, aborted myocardial infarction in a long wrap-around left anterior descending artery and microvascular dysfunction). The second one is the neuro-hormonal including blood borne catecholamine cardiac toxicity or intense sympathetic nervous system activation with local cardiac sympathetic disruption and norepinephrine spillover. Substantial literature data challenge the first hypothesis and the blood borne catecholamine cardiac toxicity [19]. Sufficient data coming mainly from neurological literature support the hypothesis that local cardiac sympathetic disruption and norepinephrine spillover causes TS [20]. This has been discussed in details elsewhere [19]. The role of beta-blockers (cardiac sympathectomy) in reducing the myocardial infarction size is well documented in the treatment of patients with ACS [39], which is a further support for the hypothesis in the present study. The findings in this study encourage that further steps in preventing and treating the cardiac sympathetic disruption in patients with ACS are justified.

\section{The limitations of the study}

The small number of patients is an inherent limitation of the study and it does not represent the whole ACS population. Furthermore, it is not certain that all PIMS have the characteristic ballooning appearance. However, this problem is also applied to the TS triggered by non-cardiac physical stress. The typical ballooning phenomenon in TS is seen only in the very acute stage of the disease and this characteristic feature will be lost as soon as the acute stage of the disease has passed [8]. A large consecutive study involving all patients with ACS with careful analysis of coronary angiography and cardiac image study including cardiac magnetic resonance examination is required. Complete resolution of the left ventricular dysfunction is one of the important characteristics of TS. However, this feature may be partially confounded by 2 other factors; the first is the ischemic myocardial necrosis which an ACS may cause; the second one ischemic injury to the sympathetic nerve in the vicinity of the diseased coronary. 


\section{Conclusion}

Twenty patients with ACS developed clinical and cardiac image features consistent with both PIMS and TS. This suggests that PIMS and TS are most probably two (among other) different names for the same acute cardiac clinical condition. ACS may trigger rather than exclude TS.

\section{References}

[1]. Braunwald E, Kloner RA (1982) The stunned myocardium: prolonged, postischemic ventricular dysfunction. Circulation 66(6): 1146-1149.

[2]. Kloner RA, Arimie RB, Kay GL, Cannom D, Matthews R, et al. (2001) Evidence for stunned myocardium in humans: a 2001 update. Coron Artery dis 12(5): 349-356

[3]. Dote K, Sato H, Tateishi H, Uchida T, Ishihara M (1991) Myocardial stunning due to simultaneous multivessel coronary spasms: a review of 5 cases. J Cardiol 21(2): 203-214.

[4]. Kawai S, Suzuki H, Yamaguchi H, Tanaka K, Sawada H, et al. (2000) Ampulla cardiomyopathy ('Takotusbo' cardiomyopathy)--reversible left ventricular dysfunction: with ST segment elevation. Jpn Circ J 64(2): 156-159.

[5]. Gianni M, Dentali F, Grandi AM, Sumner G, Hiralal R, et al. (2006) Apical ballooning syndrome or takotsubo cardiomyopathy: a systematic review. Eur Heart J 27(13): 1523-1529.

[6]. Sharkey SW, Windenburg DC, Lesser JR, Maron MS, Hauser RG, et al. (2010) Natural history and expansive clinical profile of stress (tako-tsubo) cardiomyopathy. J Am Coll Cardiol 55(4): 333-341.

[7]. Prasad A, Lerman A, Rihal CS (2008) Apical ballooning syndrome (TakoTsubo or stress cardiomyopathy): a mimic of acute myocardial infarction. Am Heart J 155(3): 408-417.

[8]. Y-Hassan S, Jernberg T (2011) Bromocriptine-induced coronary spasm caused acute coronary syndrome, which triggered its own clinical twin--Takotsubo syndrome. Cardiology 119(1): 1-6.

[9]. Y-Hassan S, Henareh L (2013) Spontaneous coronary artery dissection triggered post-ischemic myocardial stunning and takotsubo syndrome: two different names for the same condition. Cardiovasc Revasc Med 14(2): 109112.

[10]. Hirota Y, Kita Y, Tsuji R, Hanada H, Ishii K, et al. (1992) Prominent negative $\mathrm{T}$ waves with QT prolongation indicate reperfusion injury and myocardial stunning. J Cardiol 22(2-3): 325-340.

[11]. Kurisu S, Inoue I, Kawagoe T, Ishihara M, Shimatani Y, et al. (2001) Time course of electrocardiographic changes in patients with tako-tsubo syndrome: comparison with acute myocardial infarction with minimal enzymatic release. Circ J 68(1): 77-81.

[12]. Uznanska B, Plewka M, Wierzbowska-Drabik K, Chrzanowski L, Kasprzak JD (2009) Early prediction of ventricular recovery in Takotsubo syndrome using stress and contrast echocardiography. Med Sci Monitor 15(6): CS8994.

[13]. Uchida Y, Nanjo S, Fujimoto S, Yamashina S, Wagatsma K, et al. (2008) Scintigraphic studies on the etiology of Ampulla Cardiomyopathy. J Cardiol 51(2): 121-130.

[14]. Sharkey SW, Lesser JR, Zenovich AG, Maron MS, Lindberg J, et al. (2005) Acute and reversible cardiomyopathy provoked by stress in women from the United States. Circulation 111(4): 472-479.

[15]. Ibrahim T, Nekolla SG, Langwieser N, Rischpler C, Groha P, et al. (2012) Simultaneous positron emission tomography/magnetic resonance imaging identifies sustained regional abnormalities in cardiac metabolism and function in stress-induced transient midventricular ballooning syndrome: a variant of Takotsubo cardiomyopathy. Circulation 126(21): e324-326.

[16]. Nef HM, Mollmann H, Kostin S, Troidl C, Voss S, et al. (2007) Tako-Tsubo cardiomyopathy: intraindividual structural analysis in the acute phase and after functional recovery. Eur Heart J 28(20): 2456-2464.

[17]. Samuels MA (1987) Neurogenic heart disease: a unifying hypothesis. Am J Cardiol 60(18): 15J-19J.

[18]. Y-Hassan S, Yamasaki K (2013) History of takotsubo syndrome: is the syn- drome really described as a disease entity first in 1990 ? Some inaccuracies. Int J Cardiol 166(3): 736-737.

[19]. Y-Hassan S (2014) Acute cardiac sympathetic disruption in the pathogenesis of the takotsubo syndrome: A systematic review of the literature to date. Cardiovasc Revasc Med 15(1): 35-42.

[20]. Y-Hassan S (2012) Insights into the pathogenesis of takotsubo syndrome, which with persuasive reasons should be regarded as an acute cardiac sympathetic disease entity. ISRN cardiol 2012: 593735.

[21]. Sharkey SW, Lesser JR, Maron MS, Maron BJ (2011) Why not just call it tako-tsubo cardiomyopathy: a discussion of nomenclature. J Am Coll Cardiol 57(13): 1496-1497.

[22]. Iga K, Hori K, Kitaguchi K, Matsumura T, Gen H, et al. (1991) Transient segmental asynergy of the left ventricle of patients with various clinical manifestations possibly unrelated to the coronary artery disease. Jpn Circ J 55(11): 1061-1067.

[23]. Sharkey SW, Shear W, Hodges M, Herzog CA (1998) Reversible myocardial contraction abnormalities in patients with an acute noncardiac illness. Chest 114(1): 98-105.

[24]. Mizogami M, Shimo K, Taguchi T, Horita Y (2000) Stunned myocardium after hip arthroplasty. Br J Anaesth 84(4): 510-513.

[25]. Koeth O, Zeymer U, Schiele R, Zahn R (2010) Inferior ST-Elevation Myocardial Infarction Associated with Takotsubo Cardiomyopathy. Case Rep Med 1-4.

[26]. Gurlek C, van Es J, van der Burgh PH, Galjee MA, van Birgelen C (2007) Full pattern of transient apical ballooning of the left ventricle triggered by minor myocardial infarction. Neth Heart J15(9): 310-311.

[27]. Nojima Y, Kotani J (2010) Global coronary artery spasm caused takotsubo cardiomyopathy. J Am Coll Cardiol 55(9): e17.

[28]. Huczek Z, Filipiak KJ, Kochman J, Roik M, Piatkowski R, et al. (2008) Are normal coronary arteries a typical feature of apical ballooning syndrome? Am J Emerg Med 26(8): 965 e1-4.

[29]. Eitel I, Behrendt F, Schindler K, Kivelitz D, Gutberlet M, et al. (2008) Differential diagnosis of suspected apical ballooning syndrome using contrastenhanced magnetic resonance imaging. Eur Heart J 29(21): 2651-2659.

[30]. Muellerleile K, Lund G, Groth M, Barmeyer A, Sultan A, et al. (2010) Delayed-enhancement magnetic resonance imaging in patients with clinically suspected stress cardiomyopathy (Tako-tsubo). RoFo 182(1): 29-35.

[31]. Chao T, Lindsay J, Collins S, Woldeyes L, Joshi SB, et al. (2009) Can acute occlusion of the left anterior descending coronary artery produce a typical "takotsubo" left ventricular contraction pattern? Am J Cardiol 104(2): 202 204.

[32]. Lindsay J, Paixao A, Chao T, Pichard AD (2010) Pathogenesis of the Takotsubo syndrome: a unifying hypothesis. Am J Cardiol 106(9): 1360-1363.

[33]. Kono T, Morita H, Kuroiwa T, Onaka H, Takatsuka H, et al. (1994) Left ventricular wall motion abnormalities in patients with subarachnoid hemorrhage: neurogenic stunned myocardium. J Am Coll Cardiol 24(3): 636-640.

[34]. Y-Hassan S, Settergren M, Henareh L (2014) Sepsis-induced myocardial depression and takotsubo syndrome. Acute Card Care 16(3): 102-109.

[35]. Park JH, Kang SJ, Song JK, Kim HK, Lim CM, et al. (2005) Left ventricular apical ballooning due to severe physical stress in patients admitted to the medical ICU. Chest 128(1): 296-302.

[36]. Y-Hassan S (2014) Too many cooks spoil the broth: The currently existing diagnostic criteria for Takotsubo syndrome. Int J Cardiol 173(3): 568-570.

[37]. Y-Hassan S (2014) In case of strict application, the third universal definition of myocardial infarction will erase takotsubo syndrome as a diagnosis. Int J Cardiol 176(3): 1217-1219.

[38]. Prasad A, Dangas G, Srinivasan M, Yu J, Gersh BJ, et al. (2014) Incidence and angiographic characteristics of patients With apical ballooning syndrome (takotsubo/stress cardiomyopathy) in the HORIZONS-AMI trial: an analysis from a multicenter, international study of ST-elevation myocardial infarction. Catheter Cardiovasc Interv 83(3): 343-348.

[39]. Dominguez-Rodriguez A, Abreu-Gonzalez P, Reiter RJ (2014) Cardioprotection and pharmacological therapies in acute myocardial infarction: Challenges in the current era. World J cardiol 6(3): 100-106. 